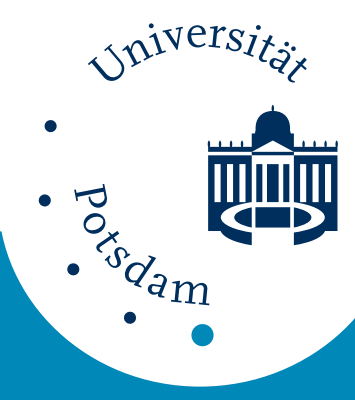

Universität Potsdam

Annette Witt, Jürgen Kurths, F. Krause, K. Fischer

On the validity of a model for the reversals of the Earth's magnetic field

NLD Preprints ; 4 


\title{
On the validity of a model for the reversals of the Earth's magnetic field
}

\author{
A. Witt ${ }^{1}$, J. Kurths ${ }^{1}$, F. Krause ${ }^{2}$, and K. Fischer ${ }^{3}$ \\ ${ }^{1}$ Arbeitsgruppe Nichtlineare Dynamik an der Universität Potsdam, Pf. 601553, \\ D-14415 Potsdam \\ ${ }^{2}$ Astrophysikalisches Institut Potsdam, An der Sternwarte, D-14482 \\ Potsdam-Babelsberg \\ ${ }^{3}$ Humboldt-Universität zu Berlin, Fachbereich Mathematik, Institut für \\ Stochastik, Psf. 1297, D-10178 Berlin
}

\begin{abstract}
We have used techniques of nonlinear dynamics to compare a special model for the reversals of the Earth's magnetic field with the observational data. Although this model is rather simple, there is no essential difference to the data by means of well-known characteristics, such as correlation function and probability distribution. Applying methods of symbolic dynamics we have found that the considered model is not able to describe the dynamical properties of the observed process. These significant differences are expressed by algorithmic complexity and Renyi information.
\end{abstract}

Keywords: reversals of Earth's magnetic field, nonlinear models, symbolic dynamics, data analysis 


\section{Introduction}

The series of reversals of the Earth's magnetic field has often been discussed as an outstanding example of complex processes. It has been generally accepted that this arrhythmic succession of reversals is an inherent signature of the geodynamo (Krause, 1984). From this viewpoint several simplified nonlinear models have been introduced to describe the large-scale time behaviour of the geomagnetic field; e.g. the two-diskdynamo (Rikitake, 1958), a system of four ordinary differential equations (Cook and Roberts, 1970),dynamo and a simple third-order recursion which may be related to a dynamo model with a back-reaction of the magnetic field to the motion in form of a third-order term (Krause and Schmidt, 1988, hereafter referred as KS).

The purpose of this contribution is to test the validity of the latter model. As has been shown by KS, it is in good accordance with the distribution of the reversals observed. Their approach compares only static properties (i.e. characterization of the distribution of reversal lengths) of the model with those data. The evolution of the Earth's magnetic field, however, is a dynamical process. Dynamical aspects can analyzed by considering the succession of reversal periods. We test here whether the model reproduces such dynamical characteristics of the process under consideration. Both the series of reversals measured and the model system are analyzed by means of techniques developed in the theory of nonlinear dynamics. Due to the short data set typical parameters in this theory, such as fractal dimensions or Lyapunov exponents, cannot be estimated reliably. Hence, we apply another concept which is based on symbolic dynamics. This leads to other characteristics, e.g. algorithmic complexity, Shannon information and Renyi information. Using these quantities we propose a $\chi^{2}$-test which allows a significant distinction of the data and the considered model from a dynamical viewpoint.

The organization of this paper is as follows: The data and the model of KS are described in section 2. The idea of symbolic dynamics and typical parameters of this approach are introduced in section 3. Section 4 presents the results of our analysis. Section 5 discusses and summarizes our findings.

\section{The data of reversals and a nonlinear model}

\subsection{The data}

We analyse here the data given by Cande and Kent (1992). Using geological techniques these authors identified 184 reversals back to $83 \mathrm{Ma}$. The accuracy of dating the time of one reversal is given as $10,000 \mathrm{a}$.

The younger data appear to be most reliable so the analysis was restricted to data for the past 40 million years (Naidu, 1971; Cox, 1981; McFadden, 1984). In particular, in the older epoch only 39 reversals were identified. 
Several authors studied the distribution of the epochs of constant polarity, i.e. the intervals between reversals, which ranges from 31,000 to 2,416,000 a. Cox (1968) was the first who found out that the distribution of the reversal history of the past 48 million years can be adequately fitted by an exponential one. Cox and several other authors have refined this idea (Naidu, 1971; Cox, 1981; McFadden, 1984). They concluded that these intervals seem to follow a gamma distribution. Basing on the second-order statistics no further structural properties of the process under consideration have been found (Laj et al., 1979, Phillips et al., 1975). It is, however, important to note that this technique only refers to a linear behaviour. In the following we also consider aspects of nonlinear dynamics.

\subsection{A nonlinear model}

Lorenz (1963) was the first to show that low-dimensional nonlinear differential equations can exhibit a complicate and rather irregular behaviour, later called deterministic chaos. Surprisingly, May (1975) found such complex structures also in the one-dimensional logistic map. Inspired by these rich structural properties of such simple equations, KS proposed to model the occurrence of the reversals by the third-order recursion

$$
x_{n+1}=a x_{n}\left(1-x_{n}^{2}\right)
$$

where the initial value $x_{0} \in[-2 / \sqrt{3},+2 / \sqrt{3}]$ is to be given and the control parameter $a$ is restricted to $a \in[0,3]$.

The main property of this deterministic difference equation (1) is that the solution $x_{n}$ changes the sign during the recursion procedure if $a>\frac{3}{2} \sqrt{3}$. KS related the sign of $x_{n}$ to the polarity of the geomagnetic field. The recursion step $n$ is equated to time as $x_{n}=x\left(t_{n}\right)=x(n \triangle t)$. Hence, eq. (1) models some kind of reversals of the geomagnetic field.

These authors found that the distribution of epoches with constant sign obtained from model (1) is in some accordance with that from the data. Hence, they determined optimum values of the control parameter $a$ and the delay time $\Delta t$ of the model by comparing the distribution of periods with constant polarity given by the data and the model.

Typical characteristics of the analyzed data are the shortest interval between reversals $l_{\min }=31,000 \mathrm{a}$ and the average length of the intervals $\bar{l}=281,000 \mathrm{a}$. The corresponding ratio $\bar{l} / l_{\min }=9.06$ is reached for $a=2.5995$ by model (1). For $\triangle t=4000$ a the distribution of both the model and the data is in a surprisingly good accordance. Therefore, $\mathrm{KS}$ argued that in view of the small amount of observational material the agreement is of sufficient quality. It should be remarked that KS fitted their model to the data of Lowrie and Alvarez (1981) and got therefore $\bar{l} / l_{\text {min }}=8.75$ and $\triangle t=5000$ a. 
It is important to note that this comparison only refers to static properties. In order to check whether or not the model is valid the dynamical properties must be included, too. In the next section we discuss how to describe the nature of a system from a dynamical viewpoint.

\section{Symbolic dynamics}

Typical parameters that characterize the dynamical properties in nonlinear systems are Lyapunov exponents and Kolmogorov entropy (Eckmann and Ruelle, 1985). It is, however, impossible to get reliable estimates of these quantities from such short series as we have in the case of the reversals identified up to now. Therefore, we take another concept into account: the symbolic dynamics (Collet and Eckmann, 1980). It is based on a coarse-graining of the dynamics, i.e. the data are transformed into a series of the same length but the elements are only a few different symbols (letters from some alphabet). This way the study of dynamics simplifies to the description of symbol sequences. In doing so one loses a great amount of detailed information, but some of the invariant, robust properties of the dynamics may be kept (Hao, 1991).

The following parameters quantify the dynamical behaviour of such a string: algorithmic complexity, Shannon information and Renyi information. We show that these techniques are efficient tools to study complex behaviour even in the case of short data sets.

\subsection{Transformations into symbolic strings}

In the following two different kinds of such transformations are considered:

\subsubsection{Natural transformation}

The data of the reversals reflect no fine structures of the amplitudes, but give only the magnetic polarity. Therefore, it is naturally to denote these amplitudes by the two symbols +1 and -1 corresponding to the two states of polarity.

For model (1) the symbol at iteration step $n$ is determined by

$$
s_{1}(n)= \begin{cases}+1 & , \text { if } x_{n}>0 \\ -1 & , \text { if } x_{n} \leq 0\end{cases}
$$

This way, we obtain symbolic descriptions of the data and the model in an equivalent manner. As explained above, we use the time step $\Delta t=4,000 \mathrm{a}$. This yields the length $n_{\max }=10,000$ of both strings, for the data and the model. 


\subsubsection{Dynamical transformations}

Next, we analyze the time intervals $l_{n}$ between the $\mathrm{n}^{\text {th }}$ and the $n+1^{\text {th }}$ reversals.

The first idea of transforming this series into a symbolic string is quite simple. One devides the interval $I$ which covers the values $l_{n}$ of this series into a finite number of parts and labels each part with a symbol. Of course, the different parts are disjunctiv.

We devide this interval $I$ into four subintervals as follows:

1. The average time difference between two adjacent reversals $\bar{l}$ splits the interval $I$ into two parts which classifies the reversal periods in short and long periods.

2. These classes are subdivided by the same procedure as above, i.e. we take the average of only the set of short (resp. long) reversal periods as a separation point.

This way, we form a symbol sequence $s_{2}(n)$ with four symbols by assigning each reversal period to the number of the corresponding subinterval.

The length of a symbol string that is based on this procedure is equal the number of reversal intervals observed. Therefore, this string consists of 143 elements, only.

Another way to introduce symbols which emphasize the dynamical aspects is the comparison of the length of adjacent reversal intervals: We investigate two variants of this special symbolic description.

Firstly, we look at pairs of lengths of adjacent reversal periods $\left(l_{n-1}, l_{n}\right)$. To each pair a symbol is assigned:

$$
s_{3}(n)=\left\{\begin{array}{lll}
1 & , \text { if } & l_{n}-l_{n+1}>0 \\
0, \text { if } & l_{n}-l_{n+1} \leq 0
\end{array}\right.
$$

The length of this symbol string determined from the observed data is equal to the number of pairs of adjacent reversal intervals, i.e. 142 elements.

Secondly, this method of symbolic description can be refined if we consider triplets instead of pairs. The resulting symbol sequence is created by:

$$
s_{4}(n)=\left\{\begin{array}{lll}
0, \text { if } & l_{n+2} \leq l_{n+1} \text { and } l_{n+2} \leq l_{n} \\
1, \text { if } & l_{n+2} \leq l_{n+1} \text { and } l_{n+2}>l_{n} \\
2, \text { if } & l_{n+2}>l_{n+1} \text { and } l_{n+2} \leq l_{n} \\
3 \text {, if } & l_{n+2}>l_{n+1} \text { and } l_{n+2}>l_{n}
\end{array}\right.
$$

Hence, the related symbolic description of the observed data consists of 141 elements.

All these transformations are performed in an analogous manner for the simulations obtained from the model.

Note, that the dynamical transformations lead in our case to short string lengths. The difficulties to analyze such strings are discussed in subsections 4.1 and 4.4. 


\subsection{Parameters for symbolic dynamics}

In this subsection we introduce typical quantities that characterize the structure of a given symbol sequence $S_{L}=\left\{s_{i}\right\}_{i=1}^{L}$, where the $s_{i}$ are elements of a finite set (alphabet) $A$. Originally, these techniques have been developed in information theory and in nonlinear dynamics. Hempelmann and Kurths (1990) showed how to apply these methods to strings with rather small length, as is typical in geophysical records.

We denote the set which contains all possible subsequences (words) $s^{k}$ of length $k$ as $A^{k} .|A|$ is the number of different letters in this alphabet $A$, called cardinality. The probability of a certain subsequence $s^{k}$ in the symbol sequence $S_{L}$ is written as $p\left(s^{k}\right)$.

The last definitions should be illustrated by a simple example: If the sequence $S_{17}=10100100100010000$ of length $L=17$ is considered the underlying alphabet $A=\{0,1\}$ has the cardinality $|A|=2$. The set which contains all words of length $2 A^{2}=\{(00)(01)(10)(11)\}$ consists of $\left|A^{2}\right|=|A|^{2}=4$ elements.

\subsubsection{Algorithmic complexity}

This measure was defined by Kolmogorov (1965) as the length of the shortest computer program which regenerates a symbol sequence studied. To make this theoretical concept operational, Lempel and Ziv (1976) proposed a procedure working with only two operations: to copy and to insert. Kaspar and Schuster (1987) suggested an algorithm that calculates a normalized measure of this kind of complexity. It works as follows: The string $S_{L}=\left\{s_{i}\right\}_{i=1}^{L}$ is divided recursively into words $w_{j}, S_{L}=\left\{w_{j}\right\}_{j=1}^{\mu}$. The words $w_{j}$ are such that $w_{1}=s_{1}$ and $w_{\alpha+1}$ is the shortest word following $w_{\alpha}$ that cannot be composed of $w_{1}, w_{2}, \ldots, w_{\alpha}$ or some part of it. Thus algorithmic complexity $c(L)$ counts the number of words of this decomposition. The algorithmic complexity $C$ is defined as the normalized number of these words

$$
C=\lim _{L \rightarrow \infty} c(L) \frac{\log _{2} L}{L} .
$$

For instance the above sequence $S_{17}=10100100100010000$ is splitted in $(1)(0)$ $(100)(1001)(000)(10000)$. This yields $c(17)=5$ and $\bar{C}=1.2022(\bar{C}$ is the estimate of $C$ from a finite string).

The algorithmic complexity $C$ characterizes symbolic strings as follows:

1. In case of periodic (prime period $m$ ) or constant symbol sequences the algorithmic complexity vanishes, because the relation $c(L) \leq m$ holds.

2. The maximum value of $C$ is taken in case of white noise: $C=\log _{2}|A|$.

3. It has been proven that for symbol sequences generated by Markov processes, algorithmic complexity agrees with Shannon entropy (Chaitin, 1987). From our experiences we find that these both quantities more generally agree. In this context 
both algorithmic complexity and Shannon entropy are measures of randomness (Wackerbauer et al., 1993).

\subsubsection{Shannon information}

Now, the symbol sequence $S_{L}$ is regarded as a set of realizations of a discrete random variable whose outputs are elements of the alphabet $A$. This leads to the Shannon information, a classical parameter of data analysis. The Shannon information of $k$-th order $H_{k}$ is defined by:

$$
H_{k}=-\sum_{s^{k} \in A^{k}, p\left(s^{k}\right)>0} p\left(s^{k}\right) \log _{2} p\left(s^{k}\right)
$$

It is easily checked that

1. $H_{k} \geq 0$, and $H_{k}=0$ only for constant symbol sequences.

2. In case of periodic symbol sequences $S$ with a prime period $m, m<k$ the Shannon information takes the value $H_{k}=\log _{2} m$.

3. The maximum of $H_{k}$ is taken if the probabilities of all words $s^{k} \in A^{k}$ are equal, then $H_{k}=k \log _{2}|A|$

This rather abstract quantity $H_{k}$ is simply to interpret: it measures the average number of bits needed to specify an arbitrary word of length $k$ in the sequence $S$.

\subsubsection{Renyi information}

The concept of Renyi information $H_{k}^{(q)}$ (Renyi, 1977) was introduced as a generalization of Shannon's ansatz:

$$
H_{k}^{(q)}=(1-q)^{-1} \log _{2}\left(\sum_{s^{k} \in A^{k}} p\left(s^{k}\right)^{q}\right)
$$

where $q$ is a real number and $q \neq 1$. It includes different averaging of probabilities. $H_{k}^{(q)}$ converges to Shannon information $H_{k}$ as $q \rightarrow 1$. The Renyi information is characterized by the following basic properties:

1. $H_{k}^{(q)}$ decreases with growing $q$.

2. If $q>1$ those words of length $k$ with large probability dominantly influence the Renyi information. This behaviour is strengthened for larger $q$ values. Vice versa if $0<q<1$ then words with small probability mainly determine the value of $H_{k}^{(q)}$.

3. In case of periodicity with prime period $m, m<k$ one gets $H_{k}^{(q)}=\log _{2} m$. If the sequence is constant $(\mathrm{m}=1)$ then $H_{k}^{(q)}=0$. 
4. If the behaviour of the sequence is such as white noise then it takes its maximum value $H_{k}^{(q)}=k \log _{2}|A|$.

The calculation of Renyi information and Shannon information for rather short time sequences leads to some difficulties. Therefore, in most cases only the information for short words $(k=1 \ldots 4)$ can be estimated with some reliability.

\section{Results of data analysis}

As expressed in the previous section, algorithmic complexity, Shannon information and Renyi information are suitable quantities to distinguish different types of structures in symbolic strings from a dynamical viewpoint. This offers an approach to check the validity of model (1) for large-scale time behaviour of the geomagnetic field.

Firstly, we must, however, study effects which may be caused by the short number of reversals documented up to now.

\subsection{Difficulties with short symbol sequences}

To investigate influences from such a small data length we perform a lot of MonteCarlo-experiments with model (1). We find that the estimated parameters depend on the initial value $x_{0}$ of this model which is, of course, unknown for the geomagnetic field. Therefore, from Monte-Carlo simulations the distributions of these parameters $C, H_{k}$ and $H_{k}^{(q)}$ are calculated and compared with the estimates obtained from the data. Our procedure consists of the following steps:

1. Simulation: Symbol sequences are generated for 1000 initial values $x_{0}$ uniformly distributed in the interval $[-2 / \sqrt{3}, 2 / \sqrt{3}]$. To get a stationary state of the dynamics the first 500 iterations are neglegted in each iteration. As the sequence length we chose that of the corresponding symbol sequence built from the data.

2. Parameter estimation: The quantities $C, H_{k}$ and $H_{k}^{(q)}(k=4$ in case of natural symbol strings and for symbol sequences built by $s_{3}, k=2$ for strings transformed via $s_{2}$ or $\left.s_{4}, q=2,4,8\right)$ are calculated for each symbol sequence generated.

3. Distribution of parameters: The histograms of the above quantities are determined. This way, we get information about the distribution of the quantities relating to the model.

4. Test of validity: The corresponding quantities obtained from the symbolic description of the data are compared with these histograms. We set up the null hypothesis: the data are compatible with the model. It is tested whether or not this hypothesis can be rejected. 


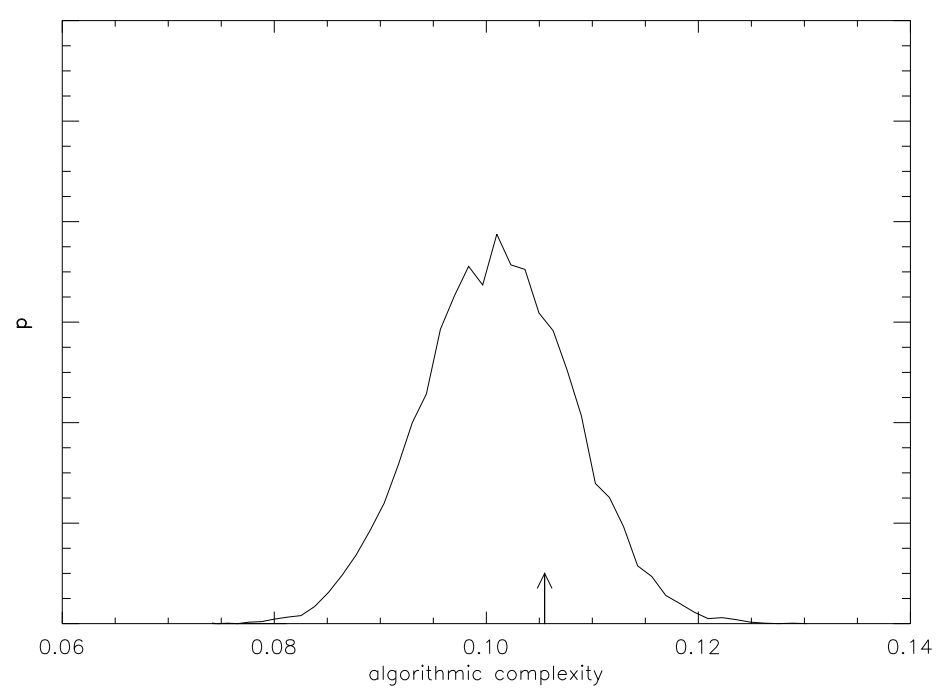

Figure 1: Distribution of algorithmic complexity for symbol strings built by rule $s_{1}$. The arrow shows the value which algorithmic complexity takes in case of the corresponding sequence from the data

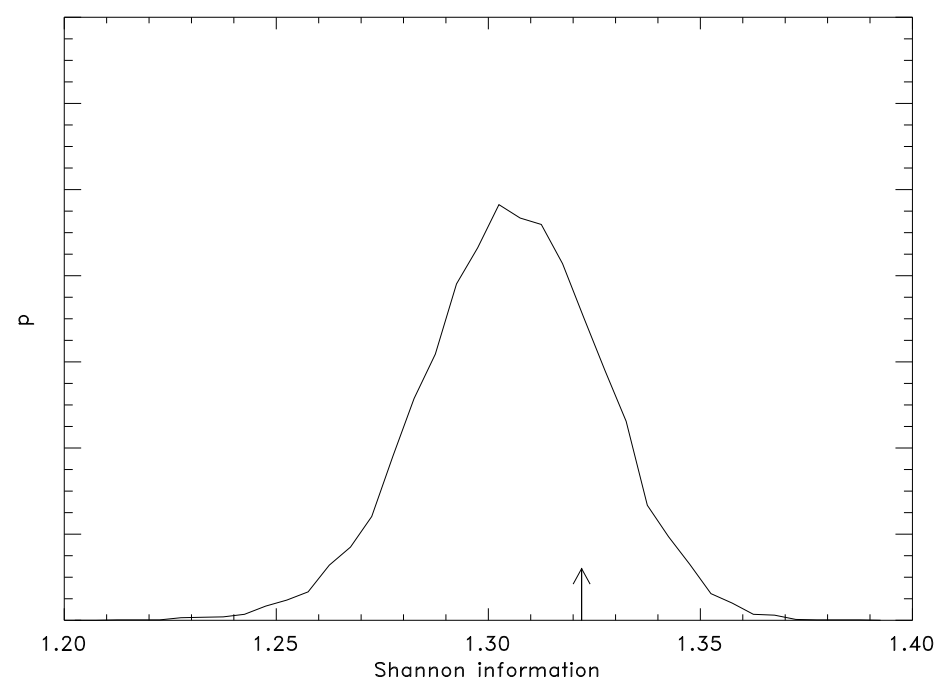

Figure 2: Shannon information $H_{4}$ for sequences built via $s_{1}$ 


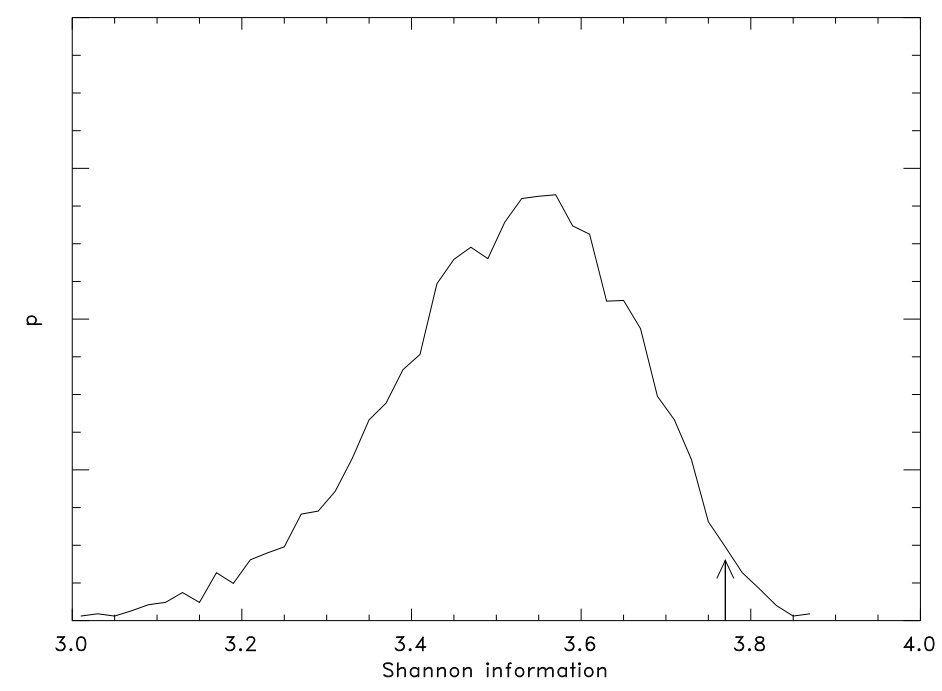

Figure 3: Shannon information $H_{2}$ for sequences built via $s_{2}$

\subsection{Analysis of strings of natural symbols}

Firstly, we analyze the strings which describe the polarity for each time $t_{n}$ (natural transformation). The histograms of algorithmic complexity $C$ and of Shannon information $H_{4}$ calculated from the symbolic description of the model appear to be similarly. Both look symetrically and one-humped distributed with mean value 0.10 and variance 0.038 for the algorithmic complexity resp. mean value 1.31 and variance 0.021 for the Shannon information.

The corresponding quantities from the observational data are $C=0.106$ resp. $H_{4}=$ 1.322. They are in a surprisingly good accordance with those from model (1) (Fig. 1,2).

We also estimate some other quantities, such as Renyi information or mutual information, and get analogous results: in every case no essential difference between the strings from the data and from the model is found.

\subsection{Analysis of strings of dynamical symbols}

Regarding the symbol strings obtained from the dynamical transformation, we emphasize the succession of different events. Thereby, the dynamical aspects of our process are placed into the foreground.

Firstly, we regard the symbolic strings describing the single durations via $s_{2}(n)$. The histogram of the Shannon information of $2^{\text {nd }}$ order $H_{2}$ is symmetrically distributed with mean value 3.53 and variance 0.15 (Fig. 3). The Shannon information calculated from the string obtained from the observational data takes the value 3.74. This is a first indication for differences between the model and the data, but there are some realizations 


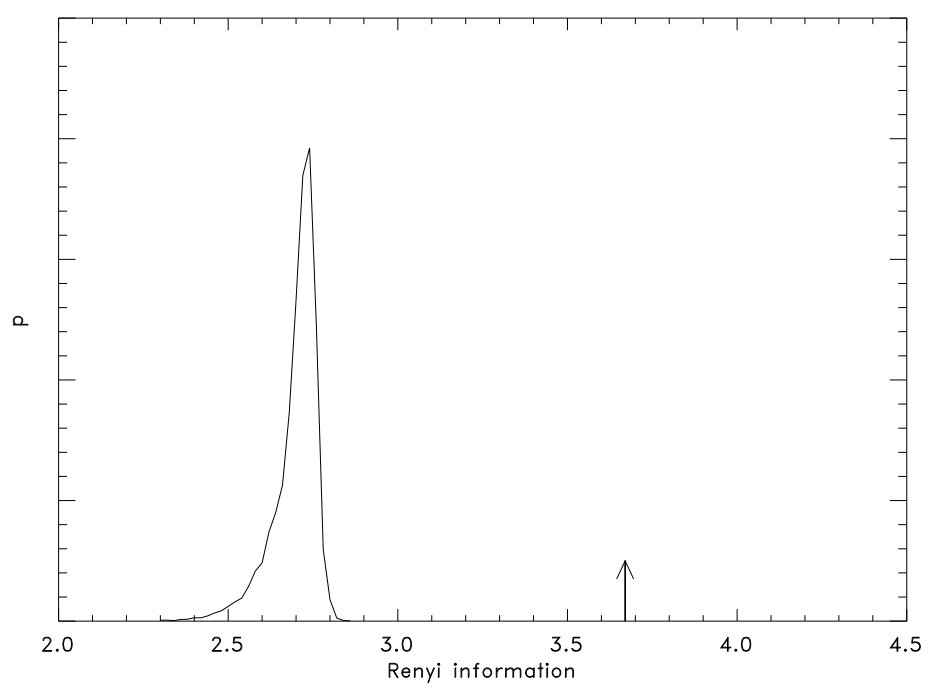

Figure 4: Renyi information $H_{4}^{(2)}$ for sequences built via $s_{3}$

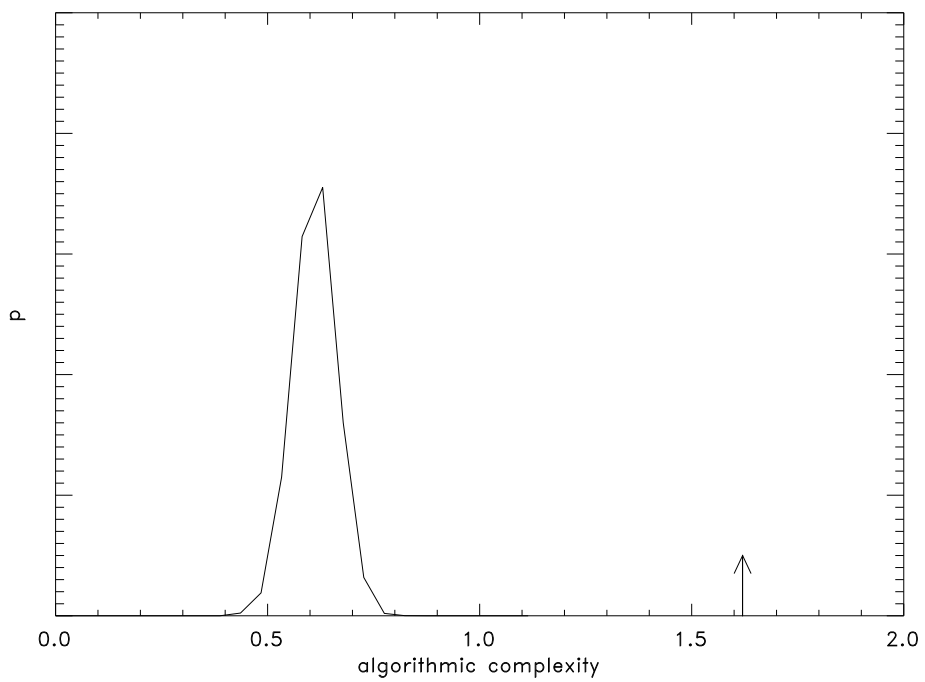

Figure 5: Algorithmic complexity $C$ for sequences built via $s_{4}$ 
of the model whose corresponding values of Shannon information are in good accordance with that from the observational record.

An opposite behaviour is found for the histogram of Renyi information $H_{2}^{q}, 2<q<8$ : These histograms are far from symmetrical in respect to the mean value. The value from the data does not fit into these histograms, it is out of the supporting interval.

Similar results are obtained if we consider the algorithmic complexity and Shannon information $\mathrm{H}_{2}$ of the other dynamically transformed symbol strings. The values from the data differ widely from the corresponding histograms (Fig 4,5).

To get a more objective result, methods from statistical inference are applied.

\section{4 $\chi^{2}$-tests for comparison the model with the data}

There is an effective tool that compares two distributions: the $\chi^{2}$-test [15]. In the following it is shown how to apply this concept to the problem considered in this paper.

The strings obtained from the data resp. the model are subdivided into small substrings of length $k(k=10,11,12)$. Now, we calculate the distribution of

1. the number of elements of a special symbol (for instance "0") in the substrings and

2. the unnormed algorithmic complexity $c(k)$ of the substrings.

Hence, we get two distributions in each case which can be compared by means of a $\chi^{2}$-test. Because the model has to provide the reference distribution it is calculated with high accuracy. We test the null hypothesis: The data are compatible with the model.

Carrying out these tests we have to coarsen the histograms built by the model in such a manner that every class contains at least 5 realizations.

This approach is performed to the three types of dynamical transformation explained in section 3.1.2. Our results are given in table 1.

Hence, we conclude that our null hypothesis has to be rejected, i.e. model (1) is not able to reproduce the dynamical properties of the observed reversals.

\section{Discussions and Conclusions}

In this paper we have used methods of nonlinear dynamics to test the validity of a model (Krause and Schmidt, 1988) for the long-time behaviour of the geomagnetic field. Although this model is only a simple third-order recursion, these authors found that it agrees in a sufficient quality with the series of reversals measured. It should be emphasized that their test of validity is based on the comparison of the distribution obtained from the reversal intervals of the model and the data. This criterion, however, refers only to static properties of systems, but the dynamical viewpoint is also essential to describe the evolution of the geomagnetic field. 
Table 1: Results of $\chi^{2}$-tests

\begin{tabular}{|c|c|c|c|}
\hline $\begin{array}{c}\text { transformation } \\
\text { into symbols }\end{array}$ & $\begin{array}{c}\text { length of } \\
\text { substrings }\end{array}$ & regarded quantity & $\begin{array}{c}\text { rejected for the } \\
\text { level of significance }\end{array}$ \\
\hline$s_{2}$ & 10 & number of symbols & $10 \%$ \\
\hline$s_{3}$ & 10 & $\begin{array}{c}\text { number of "0" } \\
c(11)\end{array}$ & $0.1 \%$ \\
& 11 & $\mathrm{c}(10)$ & $1 \%$ \\
$s_{4}$ & 10 & $\begin{array}{c}\text { number of } \\
\text { symbols }\end{array}$ & $1 \%$ \\
\hline
\end{tabular}

The main finding of our study is that this simplified model is not valid referred to dynamical characteristics of the underlying process. Due to the short data length the dynamical properties cannot be analyzed by means of well-known quantities, such as Lyapunov exponents or Kolmogorov entropy. Therefore, we have applied the concept of symbolic dynamics that leads to parameters, such as algorithmic complexity, Shannon information and Renyi information.

This viewpoint is optimally expressed if a dynamical transformation via $s_{3}$ or $s_{4}$ (eqs. 3 and 4) from the original series into a symbolic string is used. Basing on these transformations we have got a clear-cut distinction between the properties of the model and those of the reversal data. This distinction has been substantiated by means of a $\chi^{2}$-test. It yields that the null hypothesis, the data are compatible with the model, has to be rejected.

All tests are carried out for the data of Lowrie and Alvarez (1981), too. Qualitatively, the same result is found. But of course, there are some quantitative changes in the special values of the characteristice. The results of the $\chi^{2}$-tests are more sharp in case of the data of Cande and Kent (1992).

These significant differences between the data and model (1) call for a better model which does not only explain the static characteristics of the process under consideration. Surprisingly, the rejection of the rather simple model (1) requires subtle techniques that have to be based on dynamical transformations.

We have shown in this contribution that techniques from nonlinear dynamics can be effectives tools in understanding complex processes, such as long-time behaviour of the geomagnetic field. The special methods presented here seem to be of interest for the study of other systems, too. 


\section{Acknowlogment}

The authors are indepted to P. McFadden for his helpful hints and suggestions.

\section{References}

[1] Cande, S. C. and Kent, D. V., "A new geomagnetic polarity time scale for the Late Cretaceous and Cenozoic,” J. Geophys. Res. 97, 13917-13951 (1992).

[2] Chramov, A. N., Paleomagnetica, Nedra, Leningrad (1982).

[3] Collet, P. and Eckmann, J.-P., Iterated Maps on the Interval as Dynamical Systems, Birkhäuser, Bosten, Basel, Stuttgart (1980).

[4] Cook, A. E. and Roberts, P. H., "The Rikitake two-disk dynamo system," Proc. Camb. Phil. Soc 68, 547-569 (1970).

[5] Cox, A., "Length of geomagnetic polarity intervals," J. Geophys. Res. 73, 32473260 (1968).

[6] Cox, A., "A stochastic approach towards understanding the frequency and polarity bias of geomagnetic reversals," Physics of the Earth and Planetary Interiors 24, 178-190 (1981).

[7] Eckmann, J. P. and Ruelle, D., "Ergodic theory of chaos and strange attractors," Rev. Mod. Phys. 57, 617 (1985).

[8] Farmer, J. D., "Information dimension and the probalistic structure of chaos," $Z$. Naturforsch. 37a, 1304-1325 (1982).

[9] Hao, B.-L., "Symbolic dynamics and characterization of complexity," Physica D 51, 611-176 (1991).

[10] Hempelmann, A. and Kurths, J., "Dynamics of the outburst series of SS Cygni," Astron. Astrophys. 232, 356-360 (1990).

[11] Kaspar, F. and Schuster, H. G., "Easily calculable measures for the complexity of spatiotemporal patterns," Phys. Rev. A 36 (1987).

[12] Kolmogorov, A. N., "Three approaches to the quantitative definition of information," Probl. Inf. Theory 1, 3 (1965).

[13] Krause, F., "Simple nonlinear recursions and mean-field concept," Astr. Nach. 305 281-288 (1984) 
[14] Krause, F. and Schmidt, H.-J., "A low-dimensional attractor for modelling the reversals of the Earth's magnetic field," Physics of the Earth and Planetary Interiors 52, 23-29 (1988).

[15] Larson, J. H., Introduction to the theory of statistics, J. Wiley and Sons, New York (1973)

[16] Laj, C., Nordemann, D. and Pomeau, Y., "Correlation function analysis of geomagnetic field reversals," J. Geophys. research 84 4511-4515 (1979).

[17] Lempel, A. and Ziv, J., "On the complexity of finite sequences," IEEE Trans. Inf. Theory 22 75-88 (1979).

[18] Lempel, A. and Ziv, J., "Compression of individual sequences via variable-rate coding," IEEE Trans. Inf. Theory 24 530-536 (1978).

[19] Lorenz, E. N., "The problem of deducing the climate from the governing equations," Tellus, 16, 1-11 (1964).

[20] Lowrie, W. and Alvarez, W., "One hundred million years of geomagnetic polarity history," Geology 9, 392-397 (1981).

[21] May, R. M., "Simple mathematical models with very complicated dynamics," Nature 261, pp. 459. (1976)

[22] McFadden, P., "Statistical tools for the analysis of geomagnetic reversal sequences," J. Geophys. Res. 89, 3363-3372 (1984).

[23] Naidu, P., "Statistical structure of geomagnetic field reversals," J. Geophys. Res. 76, 2649-2662 (1971).

[24] Phillips, J. D., Blakely, R. J. and Cox, A., "Independence of geomegnetic polarity intervals," Geophys. J. R. Astron. Soc. 43, 747-754 (1975).

[25] Renyi, A., Wahrscheinlichkeitsrechnung. Mit einem Anhang über Informationstheorie, Deutscher Verlag der Wissenschaften, Berlin (1977).

[26] Rikitake, T. ,"Oscillations of a system of disc dynamos," Proc. Camb. phil. Soc. 54, 89-105 (1958).

[27] Schwarz, U., Kurths, J., Witt, A. and Benz, A. O., "Analysis of solar spike events," Astronomy and Astrophysics 277, 215-224 (1993).

[28] Wackerbauer, R., Witt, A., Atmanspacher, H., Kurths, J. and Scheingraber, H., "Quantification of structural and dynamical complexity," Chaos, Solitons and Fractals (in press). 